\author{
Military Technical College \\ Kobry El-Kobbah, \\ Cairo, Egypt.
}



$16^{\text {th }}$ International Conference
on Applied Mechanics and

\title{
AEROACOUSTIC RESPONSE OF ISOLATED CYLINDER(S) IN CROSS-FLOW AT DIFFERENT LOCATIONS INSIDE A RECTANGULAR DUCT
}

\author{
N. Arafa ${ }^{*}$, A. Mohany ${ }^{\dagger}$ and M. Hassan ${ }^{\ddagger}$
}

\begin{abstract}
The flow-excited acoustic resonance of single and multiple isolated cylinders in crossflow is investigated experimentally. The effect of the cylinder(s) proximity to the acoustic particle velocity nodes of the first three acoustic cross-modes is presented. During the experiments, the acoustic cross-modes of the duct housing the cylinders are selfexcited. For the case of a single cylinder, it is observed that although the cylinder's location doesn't significantly affect the process of vortex shedding, it affects the mechanism of the flow-excited acoustic resonance and the levels of the generated acoustic pressure. When the cylinder is shifted away from the acoustic particle velocity anti-node of a certain acoustic cross-mode, a combination of cross-modes is excited with intensities that seem to be proportional to the ratio of the acoustic particle velocities of these modes at the cylinder's location. For the case of two and three isolated cylinders positioned simultaneously side-by-side in the duct, it is observed that when the cylinders are positioned at different acoustic particle velocity anti-nodes of different cross-modes, the intensities of the excited acoustic resonance of these cross-modes are amplified compared to those with single cylinder. Nevertheless, when one cylinder is positioned at the acoustic particle velocity anti-node of a certain cross-mode and another cylinder is positioned at its acoustic particle velocity node, i.e. a cylinder that should excite the resonance and another one that should supress it, respectively; the excitation always takes over and the resonance occurs. Moreover, as the cylinder moves closer to the duct's wall, the Strouhal number value decreases due to the interference between the wake of the cylinder and the duct's wall. Therefore, the acoustic resonance for this case occurs at slightly higher flow velocities.
\end{abstract}

\section{KEY WORDS}

Aeroacoustics, Acoustic Excitation, Flow-excited Resonance, Side-by-side Cylinders.

PhD Candidate, University of Ontario Institute of Technology Oshawa, Ontario, Canada, Email: nadim.arafa@uoit.ca.

† Assistant Professor, University of Ontario Institute of Technology Oshawa, Ontario, Canada, Email: atef.mohany@uoit.ca.

‡ Associate Professor, University of Guelph, Guelph, Ontario, Canada, Email: mahassan@uoguelph.ca. 


\section{NOMENCLATURE}

$\begin{array}{cl}c & \text { Speed of sound [343 } \mathrm{m} / \mathrm{s}] \\ D & \text { Cylinder diameter [m] } \\ f_{a} & \text { Frequency of acoustic cross-mode } \\ f_{v} & \text { Frequency of vortex shedding [Hz] } \\ H & \text { Height of the duct [m] } \\ M & \text { Mach number } \\ n & \text { Index } \\ P^{*} & \text { Normalized acoustic pressure } \\ P_{r m s} & \text { Root mean square of acoustic } \\ S t & \text { Strouhal number } \\ U & \text { Upstream flow velocity [m/s] } \\ u & \text { Acoustic particle velocity [m/s] } \\ U_{r} & \text { Reduced velocity } \\ \rho & \left.\text { Air density [kg/m } / \mathrm{m}^{3}\right]\end{array}$

\section{INTRODUCTION}

The generation of sound due to flow over bodies or 'Aeolian tones' was first observed and documented by Strouhal in 1878 [1]. Initially, this phenomenon was attributed to the friction between the air stream and any moving body and thus referred to by 'friction tones'. Afterwards, the phenomenon became of interest for many researchers who concluded that the sound is actually a result of the periodic vortex shedding behind any bluff body [2-5]. Moreover, for a bluff body contained inside a duct, when the vortex shedding frequency coincides with one of the acoustic natural frequencies of the duct, a feedback cycle may occur where the vortex shedding acts as a sound source and excites an acoustic standing wave which, in turn, enhances the shedding process and thereby creates a strong acoustic resonance. This process is known as the flow-excited acoustic resonance and it often leads to the generation of severe noise problems and/or excessive vibrations [6]. Since the flow-excited acoustic resonance phenomenon is not yet fully understood, it can be dangerously unpredictable and may cause catastrophic failures in many engineering applications, such as tube bundles of heat exchangers. Thus, the acoustic resonance of tube bundles has received a considerable amount of attention over the past few decades [7-13]. Although different configurations and test facilities were used in these studies, all of them agreed on the occurrence of the phenomenon and the severity of the generated sound pressure levels, which exceeded $160 \mathrm{~dB}$ in some cases [11- 15]. However, due to the complex flow structure in tube bundles, several investigations have been performed in order to simplify the problem to basic flow configurations, such as a single cylinder, side-byside cylinders, and tandem cylinders, in order to understand the underlying physics of the flow-sound interaction mechanism [16-21].

Several techniques have been suggested to supress the acoustic resonance in tube bundles. One of the proposed techniques is to change the frequency of the excited acoustic mode of the system by placing baffles in the tube bundle. Basically, this technique shifts the acoustic resonance frequency above the vortex shedding frequency threshold and therefore the acoustic resonance would not occur at the 
given range of flow velocities. However, this technique is not effective to supress the higher acoustic modes [22]. Zdravkovich and Nuttall [23] investigated the suppression of the acoustic resonance in tube arrays by eliminating different cylinders from specific locations inside the tube bundle. They found that the removal of any of the cylinders that are located in the first row would eliminate the acoustic resonance. However, for the second row, only removal of the cylinders that are located at the acoustic pressure node of a certain cross-mode would be effective to suppress the acoustic resonance. Furthermore, for the third row, the removal of any cylinder did not suppress the acoustic resonance even when the cylinders that are located at the acoustic pressure node were removed. These results are quite surprising and thus, the relationship between the cylinder location inside a tube bundle and the acoustic resonance mechanism needs to be further investigated.

The acoustic cross-modes of a duct are the modes that excite acoustic standing wave inside the duct in a direction perpendicular to both the cylinder axis and the main flow velocity. These acoustic cross-modes oscillate with a simple harmonic motion in time [24]. The frequencies of the acoustic cross-modes are given by:

$f_{a, n}=n \frac{c}{2 H}, \quad n=1,2,3, \ldots$

where $n$ is an index that indicates the order of the cross-mode, $c$ is the speed of sound, and $H$ is the duct height. Figure (1) shows the distribution of both the acoustic pressure and the acoustic particle velocity along the duct height for the first three cross-modes.



Fig. 1. Theoretical Acoustic Pressure and Acoustic Particle Velocity Distribution along the Duct Height.

On the other hand, the value of the vortex shedding frequency behind a circular cylinder depends on the flow velocity and the cylinder diameter. The vortex shedding frequency is given by:

$f_{v}=S t \frac{U}{D}$

where $S t$ is the Strouhal Number, $U$ is the flow velocity, and $D$ is the diameter of the cylinder. The flow-excited acoustic resonance will occur upon the coincidence of the vortex shedding frequency with one of the acoustic cross-mode frequencies of the duct. The position of the cylinder with respect to the acoustic cross-mode's nodes or anti-nodes will dictate whether a certain cross-mode is excited or not. Therefore, the objective of this work is to investigate the effect of the relative location of a single 
cylinder within a rectangular duct on the excitation mechanism of the flow-excited acoustic resonance. Furthermore, the combined effects of positioning multiple cylinders within the duct are presented where the cylinders are positioned at the acoustic particle velocity nodes or anti-nodes for the first three acoustic cross-modes of the duct.

\section{EXPERIMENTAL SETUP}

The experimental set-up used in this work was designed so that a self-generation of acoustic resonance for the first three acoustic cross-modes of the duct housing the cylinders can be obtained. As show in Figure (2), this experimental setup consists of an open loop wind tunnel connected to a centrifugal air blower that is driven by an electrical motor equipped with a variable frequency drive to control the flow velocity in the duct. The test section is $254 \mathrm{~mm}$ high and $127 \mathrm{~mm}$ wide. This test section is connected to a single-sided diffuser with a divergence angle of $14^{\circ}$ and both of them are manufactured out of plywood with $19 \mathrm{~mm}$ thickness. The diffuser's divergence angle is selected so that the diffuser can provide a considerable recovery of pressure while maintaining minimum flow separation. The diffuser is connected to the blower via a flexible connection in order to reduce any vibration transmission to the test section. The maximum flow velocity achievable in this configuration is $160 \mathrm{~m} / \mathrm{s}$. The tested cylinders were manufactured out of industrial grade Aluminum and were rigidly mounted between the side walls of the test section where they are subjected to cross-flow air stream.



Fig. 2. Schematic of the Experimental Setup, Dimensions are in Millimeters.

The acoustic pressure is measured by means of a pressure microphone manufacture by PCB Pizotronics (model number: 377A12) and has a sensitivity of $0.254 \mathrm{mV} / \mathrm{Pa}$. The microphone is flush mounted on the top wall of the test section and firmly fixed to the wall by a Brass tube fitting and insulated with a Teflon O-ring. The output signal is acquired via a data acquisition card where a code for acquiring the signal and performing spectral analysis was prepared and tested with known signals. The sampling frequency used in all of the measurements is $10 \mathrm{kHz}$ and each signal is averaged 100 times which correspond to 100 second in real time.

Figure (3) shows the microphone position with respect to the cylinder's location and the used coordinates. The microphone is positioned at the location of the maximum acoustic pressure, which was determined in a separate experiment as shown in Figure (4). In that experiment, the microphone was flush-mounted at the top wall of the test section and moved to several stream-wise locations, with respect to the cylinder, in order to determine the location of the maximum acoustic pressure when 
the flow-excited acoustic resonance of the first acoustic cross-mode is obtained. It is shown that the position that gives the maximum pressure is around $25.4 \mathrm{~mm}$ downstream of the cylinder. All of the results presented here were obtained when the microphone is positioned at this location.



Fig. 3. Schematic Side View of the Test Section Showing the Coordinates System, the Cylinder is Positioned at $\mathrm{Y} / \mathrm{H}=0$ and $\mathrm{X} / \mathrm{H}=0$, the Microphone is Flush-Mounted on the Top Wall at $\mathrm{Y} / \mathrm{H}=0.5$ and $\mathrm{X} / \mathrm{H}=-0.1$.



Fig. 4. Peaks of the Acoustic Pressure of the First Cross-Mode versus the Microphone Positions for a Single Cylinder, $\mathrm{D}=19 \mathrm{~mm}$ and $\mathrm{U}=70.3 \mathrm{~m} / \mathrm{s}$. The Cylinder is Located at $\mathrm{Y} / \mathrm{H}=0$ and $\mathrm{X} / \mathrm{H}=0$. Negative Values Represent the Downstream Direction.

\section{RESULTS OF SINGLE CYLINDER}

The aeroacoustic response of the cylinder is obtained by performing spectral analysis and characterizing the measured acoustic pressure with respect to the flow velocity. Figure (5) shows a waterfall plot of the acoustic pressure spectra for a cylinder positioned in the middle of the duct height, $\mathrm{Y} / \mathrm{H}=0$, while Figure (6) shows the aeroacoustic response for the same case. The data points in Figure (6) are extracted from the waterfall plot of the acoustic pressure spectra where each point represents the amplitude and frequency of the vortex shedding component taken from the pressure spectrum at each flow velocity. Since the values of the vortex shedding frequency depend on the cylinder's diameter, as shown in Eqn. (2), the frequency coincidence will occur over different velocity ranges. Thus, the reduced velocity will be used to represent the flow velocity in order to avoid such variations. The reduced velocity is given by:

$U_{r}=\frac{U}{f_{a} D}$ 




Fig. 5. Waterfall Plot of the Pressure Spectra for a Single Cylinder Positioned at $\mathrm{Y} / \mathrm{H}=0, \mathrm{D}=15.7 \mathrm{~mm}$.

Figure (6) shows that the frequency of the vortex shedding progresses with the flow velocity following a linear relationship with an average Strouhal number of 0.196 . When the value of the vortex shedding frequency coincides with the frequency of the first acoustic cross-mode, acute acoustic resonance is generated. Moreover, the frequency is retrained at the value of the first acoustic cross-mode over a certain range of velocities, which is referred to by the lock-in region. As the flow velocity increases, the vortex-shedding frequency exits the lock-in region and follows the same linear relationship until reaching the frequency of the third acoustic crossmode, where another lock-in region is obtained and severe noise is generated. The second acoustic cross-mode is not excited in this case as the cylinder is located exactly at its acoustic pressure anti-node, which is an acoustic particle velocity node, as shown in Figure (1). Figure (7) shows a waterfall plot of the acoustic pressure spectra for the same cylinder positioned at $63.5 \mathrm{~mm}$ away from the duct's centerline, i.e. $\mathrm{Y} / \mathrm{H}=0.25$, while Figure (8) shows the aeroacoustic response for this case.








Fig. 6. Aeroacoustic Response of a Single Cylinder in Cross-Flow Positioned at $\mathrm{Y} / \mathrm{H}=0, \mathrm{D}=15.7 \mathrm{~mm}$.

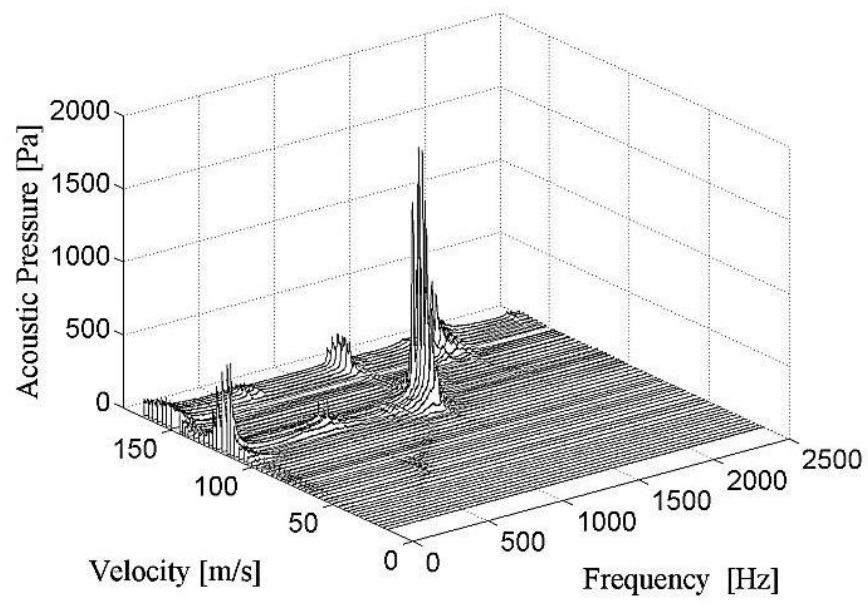

Fig. 7. Waterfall Plot of the Pressure Spectra for a Cylinder Positioned at $\mathrm{Y} / \mathrm{H}=0.25$, $\mathrm{D}=15.7 \mathrm{~mm}$.

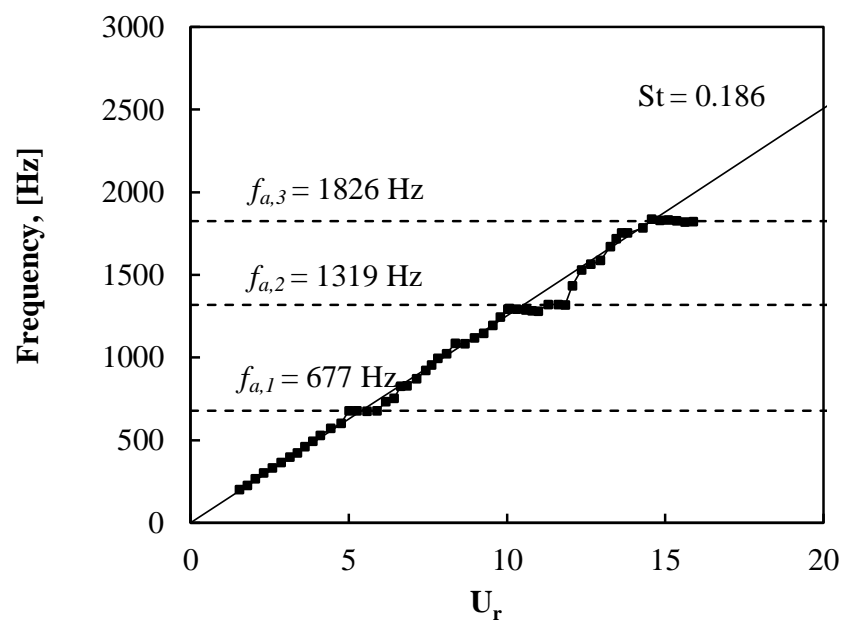






Fig. 8. Aeroacoustic Response of Single Cylinder in Cross-Flow Positioned at $\mathrm{Y} / \mathrm{H}=0.25, \mathrm{D}=15.7 \mathrm{~mm}$.

It is clear from Figure (8) that the second acoustic cross-mode excitation is dominant as the cylinder is located at its acoustic pressure node. However, the first and the third cross-modes are also excited in this case as the cylinder is not located at any of their acoustic pressure anti-nodes. The generated acoustic pressure depends on the dynamic head of the flow and thus, on the flow velocity. This means that the higher cross-modes have elevated acoustic pressure levels because they occur at higher velocities. Thus, a normalized acoustic pressure is used to eliminate the effect of the dynamic head. The normalized acoustic pressure is given by:

$P^{*}=\frac{P_{r m s}}{0.5 \rho U^{2} M}$

where $P_{r m s}$ is the root mean square amplitude of the sound pressure, $\rho$ is the fluid density, $U$ is the flow velocity, and $M$ is the Mach number. Using this normalization methodology has the advantage of unifying the comparison of the lock-in regions and collapses the normalized acoustic pressure of different cross-mode to the same order of magnitude.

Figure (9) shows a comparison of the normalized acoustic pressure for a single cylinder positioned at several vertical locations inside the duct. It is observed that when the cylinder is located in the middle of the duct height, i.e. $\mathrm{Y} / \mathrm{H}=0$, the excitation of the first and the third acoustic cross-modes is the highest among the other locations. This is due to the fact that this location is the acoustic particle velocity antinode for both the first and the third acoustic cross-modes; refer to Figure (1). Moreover, it is clear from Figure (9) that as the cylinder moves away from the duct centerline, the excitation levels of the first acoustic cross-modes, around reduced velocity value of 5 , decreases. It is also observed that as the cylinder approaches $\mathrm{Y} / \mathrm{H}=0.25$, which is the acoustic particle velocity anti-node of the second crossmode, the level of excitation of the second cross-mode increases, around reduced velocity value of 10 . Yet, this level decreases as the cylinder moves beyond this point, e.g. at $\mathrm{Y} / \mathrm{H}=0.375$. Similar results are observed for another single cylinder with a diameter of $15.7 \mathrm{~mm}$ located at the same vertical positions, as shown in Figure (10), which indicates that this behaviour is independent of the cylinder's diameter. 




Fig. 9. Comparison of the Aeroacoustic Responses of a Single Cylinder Positioned at Various Vertical Locations, $\mathrm{D}=12.7 \mathrm{~mm}$.



Fig. 10. Comparison of the Aeroacoustic Responses of a Single Cylinder Positioned at Various Vertical Locations, $\mathrm{D}=15.7 \mathrm{~mm}$.

It is clear from Figures (9) and (10) that the level of the acoustic pressure for each acoustic cross-mode depends on the cylinder's location. Since the acoustic resonance mechanism seems to be triggered by the acoustic particle velocity at the cylinder's location, it is interesting to determine if the levels of the acoustic pressure at different cylinder locations are proportional to the normalized acoustic particle velocity distribution.

Figure (11) shows the ratio of the acoustic particle velocity at the cylinder's location to the maximum acoustic particle velocity for each cross-mode. This ratio is equal to the ratio of the acoustic pressure excited at each cylinder location to the maximum acoustic pressure obtained when the cylinder is located at the acoustic particle velocity anti-node of that cross-mode. 
(a) First cross-mode

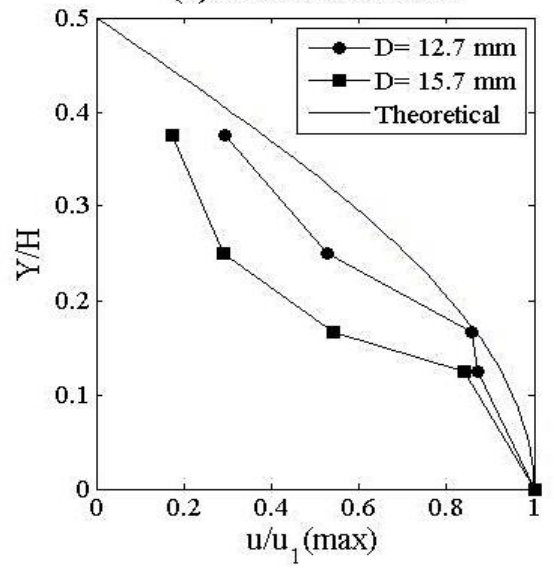

(b) Second cross-mode

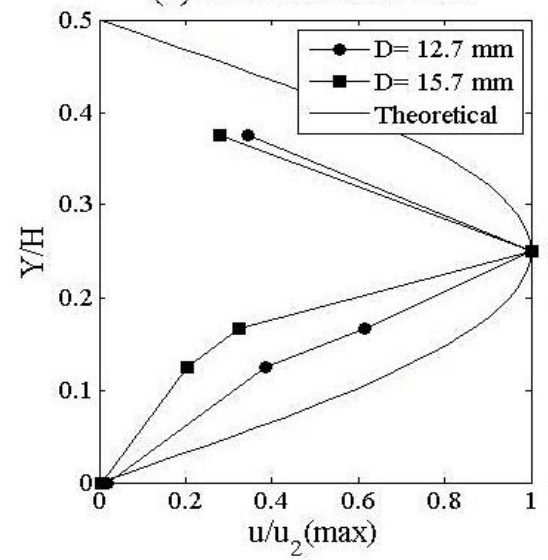

(c) Third cross-mode

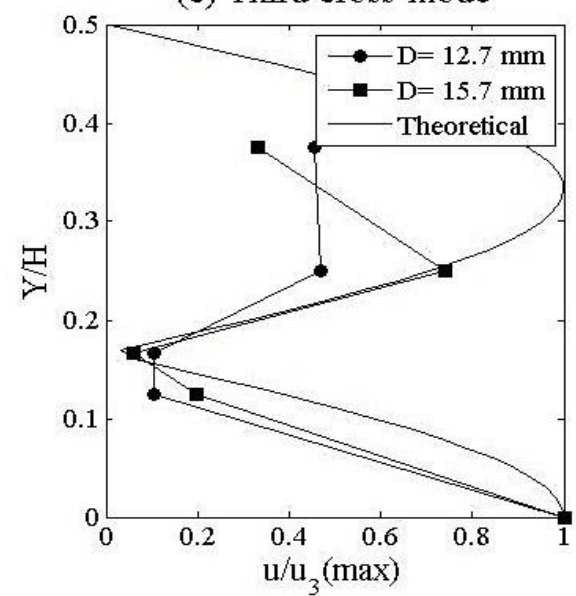

Fig.11. Distribution of the Ratio of Acoustic Particle Velocity to the Maximum Value of Acoustic Particle Velocity Along the Duct Height for (a) First, (b) Second, (c) Third Cross-Modes. The Theoretical Normalized Distribution is Shown for Each Case.

It is observed from Figure (11) that this distribution seems to follow the theoretical sinusoidal distribution of the acoustic particle velocity to some extent. This indicates that the excitation mechanism depends on the acoustic particle velocity distribution rather than the acoustic pressure distribution. It also observed that the deviation from the theoretical distribution increases as the cylinder approaches the duct's wall, which can be attributed to the interference between the duct's wall and the wake of the cylinder. It is worth to mention that the frequency values of each excited acoustic cross-mode were not constant for all the tested cases. Table (1) shows the acoustic resonance frequencies for the first three cross-modes for the tested cylinders. The variation in the resonance frequencies can be explained by the fact that the existence of the cylinder changes the path of the oscillating fluid particles, and hence the frequency will differ for different cylinder diameters. However, this effect is more pronounced for the third acoustic cross-mode, which could be the result of an added acoustic damping. Caughey and O'Kelly [25] observed similar trend for a linear dynamic system and reported that the highest natural frequency is vulnerable to decrement because of the added damping effect at higher excitation levels. 
Table 1. Frequency Values of Acoustic Cross-Modes, $D_{1}=12.7 \mathrm{~mm}$ and $D_{2}=15.7 \mathrm{~mm}$.

\begin{tabular}{|l|c|c|c|c|c|c|}
\hline \multirow{2}{*}{ Y/H } & \multicolumn{2}{|c|}{$\begin{array}{c}\text { First } \\
\text { cross-mode } \\
\text { (Hz) }\end{array}$} & \multicolumn{2}{c|}{$\begin{array}{c}\text { Second } \\
\text { cross-mode } \\
\text { (Hz) }\end{array}$} & \multicolumn{2}{c|}{$\begin{array}{c}\text { Third } \\
\text { cross-mode } \\
\text { (Hz) }\end{array}$} \\
\cline { 2 - 7 } & $\mathrm{D}_{1}$ & $\mathrm{D}_{2}$ & $\mathrm{D}_{1}$ & $\mathrm{D}_{2}$ & $\mathrm{D}_{1}$ & $\mathrm{D}_{2}$ \\
\hline $\mathbf{0}$ & 678 & 676 & $\begin{array}{c}\text { not } \\
\text { excited }\end{array}$ & $\begin{array}{c}\text { not } \\
\text { excited }\end{array}$ & 1884 & 1812 \\
\hline $\mathbf{0 . 1 2 5}$ & 680 & 676 & 1327 & 1319 & 1921 & 1855 \\
\hline $\mathbf{0 . 1 6 7}$ & 679 & 676 & 1323 & 1321 & $\begin{array}{c}\text { not } \\
\text { excited }\end{array}$ & $\begin{array}{c}\text { not } \\
\text { excited }\end{array}$ \\
\hline $\mathbf{0 . 2 5}$ & 681 & 677 & 1326 & 1319 & 1886 & 1826 \\
\hline $\mathbf{0 . 3 7 5}$ & 681 & 679 & 1324 & 1327 & 1914 & 1846 \\
\hline
\end{tabular}

Additionally, the Strouhal number values were investigated for different cylinder's locations. Rao et al. [26] have shown that the average Strouhal number value varies with the gap distance between a cylinder and a nearby wall. This Strouhal number variation affects the relationship between the frequency and flow velocity and thus may change the onset of acoustic resonance.

Figure (12) shows the variation of Strouhal number with the cylinder's locations. As the cylinder moves closer to the duct's wall, the average Strouhal number decreases due to the interference between the wake of the cylinder and the duct's wall. Therefore, the onset of acoustic resonance for such cases occurs at higher flow velocities, as shown previously in Figures (9) and (10).

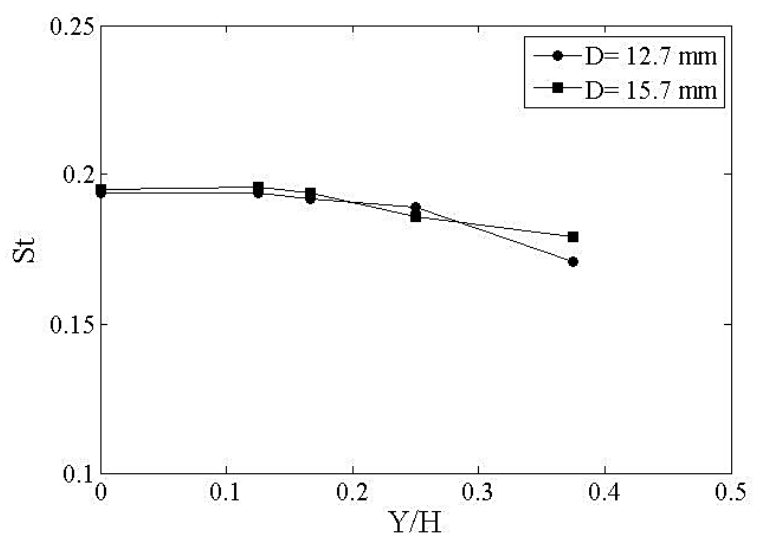

Fig. 12. Average Strouhal Number versus the Cylinder's Location.

\section{RESULTS OF MULTIPLE CYLINDERS}

The previous section shows that there is a relationship between the cylinder's location and the excitation of each acoustic cross-mode. A cylinder positioned at the acoustic particle velocity anti-node of a certain acoustic cross-mode causes an elevated excitation while, on the contrary, if the cylinder is positioned at the acoustic particle velocity node, the excitation will be supressed. One can only wonder what would happen if several cylinders are positioned simultaneously at contradicting locations. Thus, the second stage of this work is to investigate the combined effects 
of multiple cylinders positioned at different nodes or anti-nodes within the duct. For ease of comparison, a cylinder positioned at the acoustic particle velocity anti-node of a certain cross-mode will be referred to as a cylinder that excites this cross-mode, e.g. $\mathrm{Y} / \mathrm{H}=0$ for the first and third cross-modes and $\mathrm{Y} / \mathrm{H}=0.25$ for the second crossmode. On the other hand, a cylinder that is located at the acoustic particle velocity node of a certain cross-mode will be referred to as a cylinder that supresses this cross-mode.

Figure (13) shows a comparison between the aeroacoustic responses of multiple cylinders located at several positions that should excite the first three cross-modes. As mentioned before, a single cylinder positioned at $\mathrm{Y} / \mathrm{H}=0$ will excite the first and third acoustic mode while supressing the second cross-mode. Also, a cylinder positioned at $\mathrm{Y} / \mathrm{H}=0.25$ will excite the second cross-mode. However, for the case of two cylinders positioned simultaneously at $\mathrm{Y} / \mathrm{H}=0$ and $\mathrm{Y} / \mathrm{H}=0.25$, it is observed that the level of excitation is higher indicating that the presence of the cylinder at $\mathrm{Y} / \mathrm{H}=0$ enhances the effect of the cylinder positioned at $\mathrm{Y} / \mathrm{H}=0.25$ in exciting the second cross-mode and vice versa for the first and third cross-modes. For the case of two cylinders positioned simultaneously at $\mathrm{Y} / \mathrm{H}=-0.25$ and $\mathrm{Y} / \mathrm{H}=0.25$, it is observed that the level of excitation for the second cross-mode is higher indicating the additional effect of the cylinder positioned at $\mathrm{Y} / \mathrm{H}=-0.25$. However, this case did not enhance the excitation of the first cross-mode as the cylinders are not close to its acoustic particle velocity anti-node but the third cross-mode is enhanced because the cylinders are close to its acoustic particle velocity anti-nodes. Furthermore, when three cylinders were positioned simultaneously at $\mathrm{Y} / \mathrm{H}=0, \mathrm{Y} / \mathrm{H}=-0.25$, and $\mathrm{Y} / \mathrm{H}=0.25$, it is clear that the excitation levels of the three cross-modes are elevated. This indicates that the presence of the three cylinders at acoustic particle velocity antinodes enhances the acoustic excitation mechanism for all of the acoustic crossmodes and thus generates higher levels of acoustic pressure along the whole velocity range.

Figure (14) shows a comparison between the aeroacoustic responses of multiple cylinders located at other set of vertical locations within the duct. As mentioned before, a cylinder positioned at $\mathrm{Y} / \mathrm{H}=0$ will excite the first and third acoustic mode while a cylinder positioned at $\mathrm{Y} / \mathrm{H}=0.167$ will supress the third cross-mode. Thus, the purpose of this comparison is to show the combined effect of placing cylinders at contradicting locations. For example, when the two cylinders are positioned at $\mathrm{Y} / \mathrm{H}=$ 0 and $\mathrm{Y} / \mathrm{H}=0.167$, i.e. a cylinder that should excite the resonance of the third crossmode and another one that should supress it, respectively; it is observed that the excitation takes place. This indicates that the presence of the cylinder at $\mathrm{Y} / \mathrm{H}=0$ eliminates the effect of the one placed at $\mathrm{Y} / \mathrm{H}=0.167$. In fact, the presence of the latter one enhances the excitation of the first acoustic cross-mode. Additionally, when two cylinders are poisoned at $\mathrm{Y} / \mathrm{H}=-0.167$ and $\mathrm{Y} / \mathrm{H}=0.167$ simultaneously, it is observed that the excitation of the first acoustic cross-mode is further elevated. The second cross-mode is also excited in this case due to the proximity of these locations to its acoustic particle velocity anti-nodes, $\mathrm{Y} / \mathrm{H}=0.25$ and $\mathrm{Y} / \mathrm{H}=-0.25$. Furthermore, for the case of three cylinders positioned at $\mathrm{Y} / \mathrm{H}=0, \mathrm{Y} / \mathrm{H}=-0.167$, and $\mathrm{Y} / \mathrm{H}=0.167$, it is clear that the excitation levels of the first and third acoustic crossmodes are elevated while the excitation level of the second cross-mode is reduced due to the presence of the cylinder at $\mathrm{Y} / \mathrm{H}=0$. This indicates that the resonance excitation of the third cross-mode always takes over whenever a cylinder is located 




Fig. 13. Comparison of the Aeroacoustic Responses of Multiple Cylinders, $D=12.7$ $\mathrm{mm}$. Cylinders are Positioned at the Acoustic Particle Velocity Anti-Nodes.



Fig. 14. Comparison of the Aeroacoustic Responses of Multiple Cylinders, $D=12.7$ $\mathrm{mm}$. Cylinders are Positioned at the Acoustic Particle Velocity Nodes.

at its acoustic particle velocity anti-node which is similar to the second cross-mode in the cases of three cylinders positioned at $\mathrm{Y} / \mathrm{H}=0, \mathrm{Y} / \mathrm{H}=-0.25$, and $\mathrm{Y} / \mathrm{H}=0.25$. 
Figure (15) shows the noticeable shift of the normalized acoustic pressure peaks for the case of three cylinders located at $\mathrm{Y} / \mathrm{H}=0, \mathrm{Y} / \mathrm{H}=-0.167$, and $\mathrm{Y} / \mathrm{H}=0.167$ with respect to the case of three cylinders located at $\mathrm{Y} / \mathrm{H}=0, \mathrm{Y} / \mathrm{H}=-0.25$, and $\mathrm{Y} / \mathrm{H}=0.25$. This can be attributed to the interference of the cylinders' wakes as the traverse distance to diameter ratio is 3.34 in the former case compared to 5 in the latter. Zdravkovich [27] showed that the wake interference region occurs when the traverse distance to diameter is less than 3.5. Table (2) shows the frequencies of each acoustic cross-mode along with the Strouhal number of each case. It is shown that the Strouhal number for the case of three cylinders positioned at $\mathrm{Y} / \mathrm{H}=0, \mathrm{Y} / \mathrm{H}=$ 0.167 , and $\mathrm{Y} / \mathrm{H}=0.167$, where the traverse distance to diameter ratio is 3.34 , is 0.184 which explains the shift in the lock-in regions.



Fig. 15. Comparison of the Aeroacoustic Responses of Three Cylinders, $D=12.7$ $\mathrm{mm}$.

Table 2. Frequency Values of Acoustic Cross-Modes of Multiple Cylinders, $\mathrm{D}=12.7 \mathrm{~mm}$

\begin{tabular}{|l|c|c|c|c|}
\hline \multicolumn{1}{|c|}{ Y/H } & $\begin{array}{c}\text { First } \\
\text { cross- } \\
\text { mode } \\
\text { (Hz) }\end{array}$ & $\begin{array}{c}\text { Second } \\
\text { cross- } \\
\text { mode } \\
\text { (Hz) }\end{array}$ & $\begin{array}{c}\text { Third } \\
\text { cross- } \\
\text { mode } \\
\text { (Hz) }\end{array}$ & St \\
\hline $\mathbf{0}$ and $\mathbf{0 . 2 5}$ & 679 & 1332 & 1889 & 0.193 \\
\hline $\mathbf{0}$ and $\mathbf{0 . 1 6 7}$ & 679 & 1331 & 1924 & 0.189 \\
\hline $\mathbf{0 . 2 5}$ and $\mathbf{- 0 . 2 5}$ & 678 & 1324 & 1810 & 0.190 \\
\hline $\mathbf{0 . 1 6 7}$ and $\mathbf{- 0 . 1 6 7}$ & 675 & 1317 & 1806 & 0.190 \\
\hline $\mathbf{0 , 0 . 2 5}$ and -0.25 & 679 & 1332 & 1879 & 0.187 \\
\hline $\mathbf{0 , 0 . 1 6 7}$ and -0.167 & 677 & 1326 & 1926 & 0.184 \\
\hline
\end{tabular}

\section{CONCLUSIONS}

The effect of the relative location of isolated cylinder(s) within a rectangular duct on the excitation mechanism of the flow-excited acoustic resonance is investigated experimentally in this work. It has been observed that the cylinder's location affects the levels of excitation for the acoustic cross-modes of the duct. When the cylinder is 
placed at the acoustic particle velocity anti-node of a certain cross-mode, the excitation of this particular cross-mode is found to be dominant. It is also observed that as the cylinder moves away from the acoustic particle velocity anti-node of a certain cross-mode, the levels of the generated acoustic pressure decrease. This variation in the excitation levels seems to follow the theoretical sinusoidal distribution of the acoustic particle velocity which indicates that the excitation mechanism depends on the acoustic particle velocity distribution rather than the acoustic pressure distribution. Moreover, the excitation of a certain cross-mode was found to be completely supressed upon placing the cylinder exactly at the acoustic particle velocity node of this particular cross-mode. The Strouhal number is estimated for different cylinder locations and is shown to decrease as the cylinder moves away from the duct's centerline. This, in turn, shifts the onset of acoustic resonance to higher flow velocities. For the case of multiple cylinders located simultaneously at acoustic particle velocity nodes or anti-nodes within the duct, it can be concluded that the acoustic resonance of a certain cross-mode occurs whenever a cylinder is located at its acoustic particle velocity anti-node and the excitation always takes places even if another cylinder was placed at another location that should supress the resonance.

Finally, it can be concluded that the presence of a cylinder at any vertical location enhances the excitation of the first acoustic cross-mode. This is due to the fact that the first acoustic cross-mode doesn't have any acoustic particle velocity nodes within the duct height. Additionally, if the added cylinders were positioned at any of the acoustic particle velocity anti-nodes for the higher cross-modes, the levels of acoustic pressure along the whole velocity range were elevated. In one case, a shift in the lock-in regions was noticed which can be attributed to the small traverse distance to diameter ratio. These findings may be useful in explaining the complex flow-sound interaction mechanism for the case of tube bundles and they demonstrate that removing cylinders from tube bundles is not a viable technique for eliminating the acoustic resonance of the first cross-mode.

\section{ACKNOWLEDGEMENT}

The authors would like to acknowledge the financial support provided by the Natural Sciences and Engineering Research Council of Canada (NSERC).

\section{REFERENCES}

[1] Blevins, R. D., 2001. Flow-Induced Vibration, $2^{\text {nd }}$ edition. Krieger Publishing Co.

[2] Rayleigh, Lord J., 1879. "Acoustical Observation II", Philosophical magazine, 7(42), pp. $149-162$.

[3] Benard, W., 1908. "Formation of centers of circulation behind a moving obstacle", Comptes Rendus Academie des Science, 147, pp. 970 - 972, (In French, translated by Zdravkovich 1997).

[4] Relf, E. F., 1921. "On the sound emitted wires of circular section when exposed to air current", Philosophical magazine, 42(247), pp. 173 - 176.

[5] Zdravkovich, M. M., 1997. Flow around Circular Cylinders. Vol. 1: Fundamentals, $1^{\text {st }}$ edition Oxford University press. 
[6] Mohany, A., Ziada, S., 2011. "Measurements of the dynamic lift force acting on a circular cylinder in cross-flow and exposed to acoustic resonance", Journal of Fluids and Structures, 27(8), pp. 1149 - 1164.

[7] Blevins, R. D., Bressler, M. M., 1993. "Experiments on acoustic resonance in heat exchanger tube bundles", Journal of Sound and Vibration, 164(3), pp. 503 $-533$.

[8] Ziada, S., Oengoren, A. and Buhlmann, E. T., 1989. "On acoustical resonance in tube arrays: Part I. Experiments", Journal of Fluids and Structures, 3(3), pp. $293-314$.

[9] Ziada, S., and Oengoren, A., 1990. "Flow-induced acoustical resonance of inline tube bundles", Sulzer Techical Review, 1, pp. $45-47$.

[10] Ziada, S. and Oengoren, A., 1992. "Vorticity shedding and acoustic resonance in an in-line tube bundle part I: Vorticity shedding", Journal of Fluids and structures, 6(3), pp. $271-292$.

[11] Oengoren, A. and Ziada, S., 1992. "Vorticity shedding and acoustic resonance in an in-line tube bundle part II: Acoustic Resonance", Journal of Fluids and structures, 6(3), pp. $293-302$.

[12] Eisinger, F.L., Francis, J. T., and Sullivan, R.E., 1996. "Prediction of acoustic vibration in steam generator and heat exchanger tube banks", ASME Journal of Pressure Vessel Technology, 118, pp. $221-236$.

[13] Eisinger, F.L., and Sullivan, R.E., 2003. "Suppression of acoustic waves in steam generator and heat exchanger tube banks", ASME Journal of Pressure Vessel Technology, 125, pp. $221-227$.

[14] Feenstra, P.A., Weaver, D.S. and Eisinger, F.L., 2006. "A study of acoustic resonance in a staggered tube array" ASME Journal of Pressure Vessel Technology, 128, pp. $533-540$.

[15] Ziada, S., Lafon, P., 2014. "Flow-Excited Acoustic Resonance Excitation Mechanism, Design Guidelines, and Counter Measures" ASME Journal of Applied Mechanics Review, 66, p. 011002.

[16] Mohany, A., Ziada, S., 2005. "Flow-excited Acoustic Resonance of Two Tandem Cylinders in Cross-Flow", Journal of Fluids and Structures, 21, pp. 103 $-119$.

[17] Hanson, R., Mohany, A. and Ziada, S., 2009. "Flow-Excited Acoustic Resonance of Two Side-By Side Cylinders in Cross-Flow", Journal of Fluids and Structures, 25(1), pp. $80-94$.

[18] Mohany, A., Ziada, S., 2009. "A Parametric Study Of The Resonance Mechanism Of Two Tandem Cylinders In Cross-Flow", ASME Journal of Pressure Vessel Technology, 131, p. 021302.

[19] Mohany, A., Arthurs, D., Bolduc, M., Hassan, M., Ziada, S., 2013. "Numerical and Experimental Investigations of Flow-Acoustic Resonance of Side-By-Side Cylinders in a Duct". ASME Pressure Vessels \& Piping Division Conference. PVP2013-97020, Paris, France.

[20] Yang, Y., Aydin, T., Ekmecki, 2014. "Flow past tandem cylinders under forced vibration", Journal of Fluids and Structures, 44, pp. 292-309.

[21] Sumner, D., 2010. "Two circular cylinders in cross-flow: A review", Journal of Fluids and Structures, 26, pp. 849-899.

[22] Chen, S. S., 1987. Flow-induced vibration of circular cylindrical structures. Springer-Verlag, Berlin. 
[23] Zdravkovich, M.M., Nuttall, J. A., 1974. "On the elimination of Aerodynamic Noise in a Staggered Tube Bank", Journal of Sound and Vibration, 34(2), pp. $173-177$.

[24] Kinsler, L. E., Frey, A. R., Coppens, A. B., Sanders, J. V., 2000. Fundamentals of Acoustic. $4^{\text {th }}$ edition. John Wiley and Sons, Inc.

[25] Caughey T. K., O'Kelly M. E. J., 1961. "Effect of Damping on the Natural Frequencies of Linear Dynamic Systems", Journal of the Acoustical Society of America, 33(11), pp. $1458-1461$.

[26] Rao, A., Thompson, M. C., Leweke, M. C., Hourigan, K., 2013. "The flow past a circular cylinder translating at different heights above a wall", Journal of Fluids and Structures, 41, pp. $9-21$.

[27] Zdravkovich, M.M., 1985. "Flow induced oscillations of two interfering circular cylinders", Journal of Sound and Vibration, 101(4), pp. 511-521. 